\title{
Updating the Model: The Case for Independent Pharmacy to Embrace Digital Health
}

\author{
W. Kyle Cornell, PharmD/MBA Candidate 2019'; Kevin A. Clauson, PharmD²; Jeff Cain, EdD, MS ${ }^{1}$
}

${ }^{1}$ College of Pharmacy University of Kentucky; ${ }^{2}$ College of Pharmacy Lipscomb University

\begin{abstract}
Objectives: To advocate for independent pharmacy to embrace digital health as a means to improve patient outcomes and compete more strongly in an increasingly competitive business environment.

Summary: Independent pharmacies are positioned to be at the forefront of adopting digital health tools for a variety of reasons. They often can make changes to their business model faster than a major retail chain, are often in rural locations where few other providers are located, and are already starting to offer the types of clinical services that can be greatly aided by digital health. This commentary presents the case for a change in the current model of pharmacy practice to one which embraces digital health. The role of the pharmacist would grow beyond exclusively medication management to incorporate tools such as wearable health trackers and mobile phone applications. By utilizing digital health, the pharmacist can obtain a greater amount of patient health data via an asynchronous electronic uploading process, and then use that data to further improve their ability to offer clinical services.

Conclusion: Digital health is a powerful tool that should be embraced by independent pharmacy. By leveraging digital health, pharmacies can improve both accessibility and quality of care, thus providing a competitive advantage in the retail marketplace.
\end{abstract}

Keywords: Digital health, technology, independent pharmacy, community pharmacy, practice model, clinical services

\section{Challenges Facing Independent Pharmacy}

Technology allows for the provision of products and services faster than ever before. Pharmacists, who are often closer than the nearest physician's office, require no appointment, remain the most accessible healthcare professional. ${ }^{1}$ Patients, especially those with chronic conditions, reportedly visit their pharmacy as much as 1.5 to 10 times more frequently than their primary care physicians ${ }^{2}$, allowing ample opportunities for pharmacists to provide patient care.

Independent community pharmacies have long promoted their customer service as a competitive advantage over the major retail chains. ${ }^{3}$ However, they are having more difficulty competing as their bargaining power is diminished by the further growth of those chain pharmacies. Pharmacy benefit managers (PBMs) employ pricing methods and reimbursement rates that are often viewed as unpredictable and shrouded in mystery. ${ }^{4}$ Independent pharmacy, an industry that derives $92 \%$ of its sales from prescription drugs, has been particularly hurt by these trends. ${ }^{5}$ The 2017 National Community Pharmacists Association (NCPA) Digest reported a decrease in sales, prescriptions dispensed, and gross margin for the average independent pharmacy, resulting in a steady decline of independents operating across the country. ${ }^{5}$

Corresponding author: William Kyle Cornell, PharmD

College of Pharmacy University of Kentucky

$114 \mathrm{M}$ Lee T. Todd, Jr. Bldg, 789 south Limestone

Lexington, KY 40536

Email: wkco222@uky.edu
Many independent pharmacies have responded to these market pressures with innovative business models. Instead of focusing entirely on dispensing medications, they are beginning to utilize the clinical knowledge of pharmacists to offer an increased variety of services. Provision of medication therapy management, compounding services, and disease state management (e.g. diabetes, hypertension) are common ways in which independent pharmacies are creating a new competitive advantage. ${ }^{5}$ Despite independents embracing an increasingly clinical mindset, they find these services limited by an information deficit. This is evident as the majority of community pharmacies are completely disconnected from the health records possessed by the patient's other providers.

\section{Digital Health as a Potential Solution}

Digital health tools - including mobile phone applications (apps), wearable fitness trackers/monitors, and other medical devices that allow patients to track their health independently - could help address this information deficit. ${ }^{6}$ These tools produce a wealth of patient health data that is rarely utilized by providers. Leveraging these tools to access patient-generated information could grant pharmacists unprecedented levels of insight, thereby better equipping them to make informed recommendations and deliver optimal care. This commentary advocates for independent pharmacies to embrace digital health tools to alter their models of practice, thus improving clinical services and advancing their ability to compete.

In 2016 64\% of independent pharmacies utilized technology in the form of a mobile app, and increasing percentages used social media, point of sale technology, and automation in the counting and dispensing process. ${ }^{5}$ The absence of any mention 
of digital health tools, outside of mobile apps, is suggestive of the current mindset within independent pharmacy regarding their adoption. Meanwhile, chain pharmacies have begun investing in digital health in a variety of ways. Walgreens, CVS, and Rite Aid all have smartphone apps with more advanced features such as rewards programs, prescription management, and some connectivity with wearable devices. ${ }^{7}$ In 2011 Walgreens launched their Well Experience locations, which promote a redesign of the standard pharmacy layout in order to focus on digital tools and a patient-care mindset from the pharmacist. ${ }^{8}$ CVS opened their Digital Innovation Lab in 2015, which is tasked with testing digital health technologies and implementing them into their pharmacy experience. ${ }^{9}$ Most recently, in July 2018, Rite Aid announced a partnership with InTouch Health that will allow the latter to place telehealth kiosks within Rite Aid locations throughout the country. ${ }^{10}$

While chain pharmacies are largely leveraging digital health to improve the patient experience, independents may gain a twofold competitive advantage. Using these tools can further advance clinical service outcomes while also allowing for remote provision of care in rural locations that may not have a chain pharmacy or physician's office. To date, the most commonly studied use of digital health tools has been treatment of chronic disease states. Diabetes, chronic obstructive pulmonary disease (COPD), hypertension, asthma, and congestive heart failure (CHF) are among the most frequently studied. ${ }^{6}{ }^{11}$ These conditions are ideal for digital health solutions because they involve regular tracking or monitoring of parameters such as blood glucose or heart rate. ${ }^{12}$ Not only does this in align with present clinical offerings, independents also have the advantage of greater agility than their big-box counterparts when implementing major changes.

One potential selling point is that digital health tools provide a number of advantages to patients. Apps and wearable activity trackers (e.g., Apple, Fitbit, Motiv) allow patients to perform self-monitoring, which may help improve outcomes in diabetes, hypertension, and weight loss. ${ }^{13}$ Many devices also include features (e.g., medication adherence reminders, diet planning, exercise coaching, etc.) that could produce more engaged and informed patients. ${ }^{6}$ However, there is a counterintuitive disconnect between providers and patients with digital health. One study found only $20 \%$ of app users had received any recommendation from their physician to use an $\mathrm{app}^{14}$ and another that just 2 out of every 5 patients who use an app report their information to a healthcare provider. ${ }^{15}$ This communication gap is just one example of an opportunity for pharmacists to become the digital health specialist.

\section{A New Model for Independent Pharmacy Practice}

This commentary advocates for a new model of independent pharmacy practice which, through leveraging digital health tools to acquire currently unavailable patient health information, would transform the ability of these pharmacies to offer ambulatory care. Pharmacy-based diabetes management clinics provide an example of a service that could benefit from incorporation of digital health. A traditional model might consist of a new patient presenting to the clinic obtaining new medications, getting their blood glucose level checked, and receiving disease management counseling from a pharmacist. The service is focused largely on medication management and the only patient health data available to the pharmacy is that obtained during each visit.

Some healthcare professionals have begun suggesting that providers, and specifically pharmacists, should transition their role into that of healthcare coach. ${ }^{15,16}$ The job must include a focus on equipping patients with the tools - medications, counseling, or a wearable activity tracker - to live a healthy life in all facets. Martin et al have suggested a model in which the pharmacist frequently receives patient vitals and other data through a mobile-sourced information system. ${ }^{6}$ The pharmacist would be alerted to any abnormal values, and could employ artificial intelligence-facilitated triaging. This process would culminate with a follow-up appointment or videoconference with the patient, and other relevant providers, to discuss the results and make any necessary changes to the care plan. ${ }^{6}$

A new model of independent pharmacy practice would combine the idea of digital health coaching, the model proposed by Martin et al, and collaborative practice agreements to provide expanded clinical services. This model that leverages digital health tools would encompass all facets of equipping patients to live a healthy lifestyle. The pharmacy would no longer just sell the patient at their clinic diabetes medications and a blood glucose monitor. It would also stock wearable activity trackers and other monitors. In addition to providing medication and diet counseling, the pharmacist would inform the patient about which mobile apps can be used to plan their diet, track calories, improve medication adherence, and upload their daily blood glucose readings. Pharmacies could simultaneously launch a digital healthenhanced diabetes prevention program for those patients with prediabetes as a means to generate a new stream of revenue.

Regardless of focus, the pharmacy must have its own app or internet portal which connects to third-party apps and allows real-time data uploads to the patient's records for the pharmacist to review. The patient would schedule follow-up visits at extended intervals, with more immediate appointments to occur if the pharmacist is alerted to abnormal readings from the patient's data. During clinic visits the pharmacist still provides medication expertise but can also explain the health data to the patient, provide nonpharmacological and digital health counseling to improve lifestyles, and use collaborative practice agreements to make medication changes without needing to consult a physician. Utilizing digital health empowers the pharmacist to undertake clinical practice that goes beyond medication dispensing and counseling, while simultaneously granting patients greater access to more convenient high-level care. 


\section{Do Digital Health Tools Work?}

Like most innovations, implementing a digital health model is not without challenges. As much promise as these possibilities hold, they have little value if the digital health tools themselves are ineffective. Marcolino et al published a systematic review of 23 other systematic reviews, which included 371 studies and 79,665 patients in total, to assess the effectiveness of digital health interventions. The studies included a wide range of interventions, with the results presented according to disease state. Overall, digital health interventions resulted in positive outcomes for asthma, cardiac rehabilitation, congestive heart failure, chronic lung disease, diabetes, obesity, HIV management, and smoking cessation. Specific outcomes are listed in Table 1. The review concluded that evidence showed that using digital health tools in chronic disease states has benefits. ${ }^{11}$ Although the Marcolino et al study showed mixed results regarding the improvement of blood pressure in hypertensive patients, another study published in June 2018 has shown significant improvement in medication adherence with a digital health intervention. ${ }^{14}$

Other systematic reviews have shown that, when combined with behavioral change techniques, wearable activity trackers can improve health behaviors, increase physical activity, lower body mass index, and decrease blood pressure. ${ }^{17,18}$ Additional studies have determined that mainstream wearables on the market today are highly accurate in measuring heart rate, sleep duration, number of steps, distance traveled, and energy consumption. ${ }^{18}$ Speier et al found adherence rates of $90 \%$ for wearable trackers if patients are asked to use just one device with high utility. ${ }^{19}$ Overall, the evidence suggests that digital health tools can accurately monitor patient vitals as well as influence positive clinical outcomes. Advances in technology are also likely to continue making these tools more accurate and reliable.

\section{Obstacles to Overcome}

Facilitating any change this significant is difficult, so pharmacists must first be able to articulate the value of these digital health tools and provide a compelling enough case for patients and providers alike to adopt and benefit from their consistent use. The amount of options on the market can be overwhelming. Patients often are unable to differentiate between apps or devices, having only online reviews to guide them. ${ }^{16}$ Patients also suffer from device fatigue if asked to use too many wearables or apps, and will quit using them altogether. ${ }^{13,15}$ The pharmacist must embrace a new role and be prepared to inform and guide patients regarding which options are reliable, accurate, and effective. This means it is imperative that pharmacists receive adequate education on digital health tools.

Limitations remain as to what data can actually be gathered by the devices currently available. The utility of digital health tools is restricted to disease states that require monitoring of parameters in alignment with capabilities of these devices. As pharmacies continue migrating toward a more service-based model of practice with an emphasis on outcomes, billing for services will continue to be challenging. Advocating for provider status from the Centers for Medicare and Medicaid Services (CMS) to alleviate this issue must continue to be a priority for pharmacists. Finally, all healthcare professionals must perceive value in the digital health model of pharmacy for it to be successful. Unfortunately, the current lack of evidence-based guidelines gives many providers pause when considering the use of digital health. ${ }^{16}$ In order to optimize patient care, it is important that other professions respect the recommendations from the pharmacy regarding digital health. A convincing case for widespread acceptance is dependent on pharmacies to deliver proof of successful outcomes resulting from these practices. These obstacles and recommendations are summarized in Table 2.

\section{Conclusion}

In recent years, technological advances have shifted the way entire industries operate. The healthcare industry can be slow to embrace new technological trends. However, as the amount of digital health tools increase, there is an opportunity for radical changes in healthcare provision. Research indicates that apps and activity tracking devices are capable of improving patient health and behaviors. Pharmacists, as the most accessible provider with the least amount of information, are positioned to uniquely benefit from the adoption of digital health tools into their practice. Independent pharmacies should leverage digital health to expand their clinical services, thus optimizing outcomes and creating a competitive advantage in the market.

\section{Acknowledgements: None \\ Funding/Support: None \\ Conflicts of Interest: None}

\section{References}

1. Kelling SE. Exploring accessibility of community pharmacy services. Inov Pharm. 2015; 6(3): Article 210.

2. Tsuyuki RT, Beahm NP, Okada H, Al Hamarneh YN. Pharmacists as accessible primary health care providers: review of the evidence. Can Pharm J. 2018; 151(1): 4-5.

3. McGee JE, Peterson M. Toward the development of measures of distinctive competencies among small independent retailers. J Small Bus Manag. 2000; 38(2): 19-33.

4. Matalavage M. Navigating the complexities of pharmacy reimbursement: will we ever reach transparency? Available at https://www.pharmacytimes.com/publications/issue /2016/march2016/navigating-the-complexities-ofpharmacy-reimbursement-will-we-ever-reachtransparency. Accessed August 13, 2018. 
5. Michos L, Holmes E, Lewis R, et al. 2017 NCPA Digest. Available at ncpanet.org. Accessed August 13, 2018.

6. Martin A, Brummond P, Vlasimsky T, et al. The evolving frontier of digital health: opportunities for pharmacists on the horizon. Hospital Pharmacy. 2018; 53(1): 7-11.

7. Moukheiber Z. Notes on the transformation of Walgreens. Available at https://www.forbes.com/sites/zinamoukheiber/2013 /06/13/notes-on-the-transformation-ofwalgreens/\#367504103c1b. Accessed August 13, 2018.

8. Bringing back the neighborhood pharmacy. Available at https://www.ideo.com/case-study/bringing-backthe-neighborhood-pharmacy. Accessed August 13, 2018.

9. CVS health opens digital innovation lab in Boston. Available at https://cvshealth.com/newsroom/pressreleases/cvs-health-opens-digital-innovation-labboston. Accessed September 3, 2018.

10. Rite Aid eyes telehealth kiosks in new deal with InTouch Health. Available at https://mhealthintelligence.com/news/rite-aid-eyestelehealth-kiosks-in-new-deal-with-intouch-health. Accessed September 3, 2018.

11. Marcolino MS, Oliveira JAQ, D'Agostino $M$, et al. The impact of mhealth interventions: systematic review of systematic reviews. JMIR Mhealth Uhealth. 2018; 6(1): e23.

12. Robbins $R$, Krebs $P$, Jagannathan $R$, et al. Health app use among US mobile phone users: analysis of trends by chronic disease status. JMIR Mhealth Uhealth. 2017; 5(12): e197.
13. Shaw RJ, Steinberg DM, Bonnet J, et al. Mobile health devices: will patients actually use them? Journal of the American Medical Informatics Association. 2016; 23(3): 462-466.

14. Morawski K, Ghazinouri R, Krumme A, et al. Association of a smartphone application with medication adherence and blood pressure control: the MedISAFE-BP randomized clinical trial. JAMA Intern Med. 2018; 178(6): 802-809.

15. Ng V. Digital health coach: the evolving role of pharmacists. Available at https://www.telushealth.co/item/digital-healthcoach-evolving-role-pharmacists/. Accessed August 13, 2018.

16. Loiselle CG, Ahmed S. Is connected health contributing to a healthier population? J Med Internet Res. 2017; 19(11): e386.

17. Abedtash $\mathrm{H}, \mathrm{Holden}$ RJ. Systematic review of the effectiveness of health-related behavioral interventions using portable activity sensing devices (PASDs). J Am Med Inform Assoc. 2017; 24(5): 10021013.

18. Xie J, Wen D, Liang L, et al. Evaluating the validity of current mainstream wearable devices in fitness tracking under various physical activities: comparative ttudy. JMIR Mhealth Uhealth 2018; 6(4): e94.

19. Speier W, Dzubur E, Zide M, et al. Evaluating utility and compliance in a patient-based eHealth study using continuous-time heart rate and activity trackers. J Am Med Inform Assoc. 2018; 0(0): 1-6. 
Table 1: Condition-specific outcomes with digital health interventions ${ }^{11}$

\begin{tabular}{|c|c|}
\hline Condition & Outcomes \\
\hline Asthma & $\begin{array}{l}\text { - Improvements in cough and nighttime symptoms } \\
\text { - Decreased daily doses of medication } \\
\text { - Improvement in pulmonary function and health-related quality of life } \\
\text { - Decreased unscheduled visits to emergency departments and } \\
\text { hospitalizations }\end{array}$ \\
\hline Cardiac Rehabilitation & $\begin{array}{l}\text { - Improved exercise capacity as shown by increased distance during a } \\
\text { 6-minute walk test ( } 6 \mathrm{MWT} \text { ) } \\
\text { - More likely to achieve goals for blood pressure and body mass index } \\
\text { (BMI) }\end{array}$ \\
\hline Congestive Heart Failure & $\begin{array}{l}\text { - } \quad \text { Fewer symptom complaints } \\
\text { - Relative risk reduction of death or hospitalization }\end{array}$ \\
\hline Chronic Lung Diseases & $\begin{array}{l}\text { - Improved cough symptoms and sleep quality } \\
\text { - Increased walking distance during exercise }\end{array}$ \\
\hline Diabetes & $\begin{array}{l}\text { - Improved } \mathrm{HbA}_{1 c} \text {, low-density lipoprotein (LDL) cholesterol, and } \\
\text { microalbuminuria } \\
\text { - } \\
\text { - } \quad \text { Incruced fasting plasma glucose and 2-hour postprandial glucose } \\
\end{array}$ \\
\hline HIV Management & $\begin{array}{l}\text { - } \quad \text { Reduced viral load } \\
\text { - Increased adherence with medication taking among memory- } \\
\text { impaired patients }\end{array}$ \\
\hline Hypertension & - Mixed results regarding improvement in blood pressure \\
\hline $\begin{array}{l}\text { Overweight and Obese Adults } \\
\text { (BMI: 25-39.9) }\end{array}$ & $\begin{array}{l}\text { - } \quad \text { Short-term weight loss } \\
\text { - Improvement in percent of body fat lost } \\
\text { - Unchanged BMI and blood pressure }\end{array}$ \\
\hline Smoking Cessation & $\begin{array}{l}\text { - Increased abstinence rates at } 26 \text { weeks } \\
\text { - Increased rate of biochemically verified smoking cessation at } 6 \\
\text { months }\end{array}$ \\
\hline
\end{tabular}


Table 2: Barriers to digital health and recommendations for pharmacists

\begin{tabular}{|c|c|}
\hline Barrier & Recommendation \\
\hline $\begin{array}{l}\text { A lack of knowledge and reliable information regarding } \\
\text { digital health options causing patients to feel } \\
\text { overwhelmed, coupled the potential for device } \\
\text { fatigue. }{ }^{13,15,16}\end{array}$ & $\begin{array}{l}\text { The pharmacist must embrace a new role as the } \\
\text { knowledgeable source on digital health tools, and } \\
\text { therefore must self-educate or receive adequate } \\
\text { education on this topic. }\end{array}$ \\
\hline $\begin{array}{l}\text { Capabilities of current devices limiting what types of } \\
\text { data can be accurately obtained. }\end{array}$ & $\begin{array}{l}\text { Pharmacies must incorporate digital health tools into } \\
\text { services that align with the capabilities of current } \\
\text { technology (e.g. management of conditions referenced in } \\
\text { Table 1). }\end{array}$ \\
\hline $\begin{array}{l}\text { Challenges in receiving reimbursement from payer such } \\
\text { as CMS for clinical services provided by pharmacists. }\end{array}$ & $\begin{array}{l}\text { Pharmacists must continue advocacy efforts focusing on } \\
\text { legislation that recognizes the profession with provider } \\
\text { status, or creates programs that provide reimbursement } \\
\text { for specific services, such as the Diabetes Prevention } \\
\text { Program (DPP). }\end{array}$ \\
\hline $\begin{array}{l}\text { A lack of awareness and evidence-based guidelines for } \\
\text { the use of digital health tools preventing buy-in from } \\
\text { other providers. }{ }^{16}\end{array}$ & $\begin{array}{l}\text { Pharmacies implementing digital health tools into their } \\
\text { practice must provide evidence of positive outcomes to } \\
\text { demonstrate their effectiveness. }\end{array}$ \\
\hline
\end{tabular}

